PROCEEDINGS OF THE

AMERICAN MATHEMATICAL SOCIETY

Volume 138, Number 10, October 2010, Pages 3603-3608

S 0002-9939(10)10367-0

Article electronically published on April 14, 2010

\title{
SOME REMARKS ON HARTOGS' EXTENSION LEMMA
}

\author{
MIRAN ČERNE AND MANUEL FLORES
}

(Communicated by Franc Forstneric)

Dedicated to Professor J. M. Méndez on the occasion of his 60th birthday

\begin{abstract}
Motivated by a result and a question by E. M. Chirka we consider the Hartogs' extension property for some connected sets in $\mathbb{C}^{2}$ of the form $K=\Sigma \cup(\partial \Delta \times \bar{\Delta})$, where $\Sigma$ is a possibly nonconnected compact subset of $\bar{\Delta} \times \bar{\Delta} \subset \mathbb{C}^{2}$.
\end{abstract}

\section{INTRODUCTION}

Let $\Delta(0,1)=\Delta \subseteq \mathbb{C}$ be the unit disc and let $f$ be a holomorphic function in a connected neighbourhood of the set $K=(\bar{\Delta} \times\{0\}) \cup(\partial \Delta \times \bar{\Delta})$ in $\mathbb{C}^{2}$. It is a wellknown result by Hartogs [10] that $f$ extends holomorphically to a neighbourhood of $\bar{\Delta} \times \bar{\Delta}$. It is also classical, and an easy consequence of the Continuity Principle, that Hartogs' lemma holds if in $K$ the set $\bar{\Delta} \times\{0\}$ is replaced by the graph of a holomorphic function $\varphi: \Delta \rightarrow \Delta$ or even a finitely sheeted analytic variety $\Sigma \subseteq \bar{\Delta} \times \bar{\Delta}$ over $\Delta$.

In [4] (see also [1, 5, 9]) Chirka, using an idea from [8, proved that the same result holds if $\varphi$ is merely continuous. Although the higher dimensional version of Hartogs' lemma still holds, the higher dimensional analog of Chirka's theorem in which one considers the graph of two or more continuous functions on $\bar{\Delta}$ in general does not hold. Rosay [1] constructed two smooth functions $\varphi_{j}: \bar{\Delta} \rightarrow \bar{\Delta}$, $j=1,2$, such that there exists a connected pseudoconvex neighbourhood of the set $K=\left\{\left(z, \varphi_{1}(z), \varphi_{2}(z)\right) ; z \in \bar{\Delta}\right\} \cup\left(\partial \Delta \times \bar{\Delta}^{2}\right)$ which does not contain $\bar{\Delta}^{3}$. For a result in a positive direction, see [2, but the class of functions $\varphi_{j}$ for which the extension property holds is quite restrictive. Let us mention that these results are also related to the results on the envelope of holomorphy of a neighbourhood of a real surface in a complex surface, 7, 8, 9.

Received by the editors October 7, 2009 and, in revised form, December 17, 2009 and January 1, 2010 .

2010 Mathematics Subject Classification. Primary 32D10; Secondary 32Q60, 32Q65.

Key words and phrases. Analytic continuation, Hartogs' extension.

The first author was supported in part by grant Analiza in geometrija P1-0291 from the Ministry of Higher Education, Science and Technology of the Republic of Slovenia.

The second author was supported in part by a grant from Ministerio de Ciencia y Tecnología, MTM 2007/65009.

(C)2010 American Mathematical Society Reverts to public domain 28 years from publication 
Let $K \subseteq \bar{\Delta} \times \bar{\Delta}$ be a compact set. An obvious necessary condition that every function holomorphic in a neighbourhood of $K$ extends holomorphically to a neighbourhood of $\bar{\Delta} \times \bar{\Delta}$ is that the rational hull of $K$ equals $\bar{\Delta} \times \bar{\Delta}$. In this context Chirka [4] asked the following.

Question (Chirka). Let $K \subset \bar{\Delta} \times \bar{\Delta}$ be a connected compact set containing $\partial \Delta \times \bar{\Delta}$ and such that its rationally convex hull coincides with $\bar{\Delta} \times \bar{\Delta}$. Is it true that every function holomorphic in a connected neighbourhood of $K$ extends holomorphically to $\bar{\Delta} \times \bar{\Delta}$ ?

Motivated by this question, we consider the following generalizations of Hartogs' extension lemma.

Theorem 1. For $\varepsilon>0$, let $\Sigma_{\varepsilon}$ be given by

$$
\Sigma_{\varepsilon}=\{(z, \varepsilon) \in \bar{\Delta} \times \mathbb{C} ; \operatorname{Re} z \geq-1 / 2\} \cup\{(z,-\varepsilon) \in \Delta \times \mathbb{C} ; \operatorname{Re} z \leq 1 / 2\} .
$$

Then there exist constants $\gamma>1$ and $0<\varepsilon_{0}<1$ such that for any $0<\varepsilon<\varepsilon_{0}$, any holomorphic function in a connected $\varepsilon^{\gamma}$-neighbourhood (in the $w$-direction) of $K_{\varepsilon}=\Sigma_{\varepsilon} \cup(\partial \Delta \times \bar{\Delta})$ extends holomorphically to a neighbourhood of $\bar{\Delta} \times \bar{\Delta}$.

Observe that the $\Sigma_{\varepsilon}$-part of the set $K_{\varepsilon}$ is not connected and that for each connected component of $\Sigma_{\varepsilon}$ one cannot directly use any of the previous theorems.

An $\varepsilon^{\gamma}$-neighbourhood of $K_{\varepsilon}$ in the $w$-direction is the set

$$
\bigcup_{(z, w) \in K_{\varepsilon}}\left(\Delta(z, \delta) \times \Delta\left(w, \varepsilon^{\gamma}\right)\right)
$$

for some $\delta>0$.

As a first example to look at, Chirka considered the set $K_{\varepsilon}$, which satisfies the assumption on its rational convex hull in the question for $\varepsilon>0$ small enough. Nevertheless, in a private communication, J. P. Rosay has pointed out to us that the answer to Chirka's question is negative. An example is $K_{\varepsilon}$ itself for some small enough neighbourhood. Note that in Theorem 1 we tacitly assume that the neighborhood from which we have the extension must be 'fat' enough near the 'faces' of $\Sigma_{\varepsilon}$ as specified in the hypothesis.

While the case where $\Sigma$ is not connected might be a 'natural' place to look for a counterexample to Chirka's question, this does not seem to be the case for 'continuous' varieties over $\bar{\Delta}$.

Problem. Let $a_{0}, \ldots, a_{n-1}$ be continuous functions on $\bar{\Delta}$ such that the set

$$
\Sigma=\left\{(z, w) \in \bar{\Delta} \times \mathbb{C} ; w^{n}+a_{n-1}(z) w^{n-1}+\cdots+a_{0}(z)=0\right\}
$$

is a subset of $\bar{\Delta} \times \bar{\Delta}$. Does every function holomorphic in a connected neighbourhood $\Omega$ of the set $K=\Sigma \cup(\partial \Delta \times \bar{\Delta})$ such that $\bar{\Omega} \cap(\bar{\Delta} \times \bar{\Delta})$ is connected extend holomorphically to a neighbourhood of the bidisc $\bar{\Delta} \times \bar{\Delta}$ ?

In this direction we have the following result.

Theorem 2. Let $a_{0}, \ldots, a_{n-1}$ be continuous functions on $\bar{\Delta}$ and let

$$
\Sigma=\left\{(z, w) \in \bar{\Delta} \times \mathbb{C} ; w^{n}+a_{n-1}(z) w^{n-1}+\cdots+a_{0}(z)=0\right\}
$$

be a continuous variety over $\bar{\Delta}$. Then every function holomorphic in a connected neighbourhood of the set $K=\Sigma \cup(\partial \Delta \times \mathbb{C})$ extends holomorphically to a neighbourhood of $\bar{\Delta} \times \mathbb{C}$. 
The assumptions in Theorem 2 are much stronger than those in the Problem; that is, we assume holomorphicity on a neighbourhood of a continuous variety $\Sigma$ and the infinite cylinder $\partial \Delta \times \mathbb{C}$ to conclude holomorphic extendability into $\bar{\Delta} \times \mathbb{C}$.

\section{Proof of Theorem 1}

Let $\Omega$ be a connected neighborhood of $K_{\varepsilon}$ and $f$ a holomorphic function in $\Omega$. Since $K_{\varepsilon}$ is invariant under the transformation $(z, w) \mapsto(-z,-w)$, shrinking $\Omega$ if necessary, we may assume that $\Omega$ is invariant under such a transformation as well and hence $f$ can be decomposed into its even and odd parts. Therefore, to extend $f$, we can further assume that $f$ is even (for the odd part the reasoning being the same).

Let $\rho^{ \pm}$, defined in a smooth neighborhood $D^{ \pm}$of $\pm \operatorname{Re} z \geq-1 / 2$ and $|z| \leq 1$, be the radius of convergence of the Taylor expansion of $f$,

$$
f(z, \pm \varepsilon+w)=\sum_{n=0}^{\infty} a_{n}^{ \pm}(z) w^{n}
$$

respectively, that is,

$$
\log \frac{1}{\rho^{ \pm}(z)}=\limsup _{n \rightarrow \infty} \frac{1}{n} \log \left|a_{n}^{ \pm}(z)\right| .
$$

Since $f$ is holomorphic and even in $\Omega$, the functions $u^{ \pm}:=\log \frac{1}{\rho^{ \pm}}$are subharmonic where defined, and moreover $u^{+}(-z)=u^{-}(z)$ if $\operatorname{Re} z \leq 1 / 2$ and $|z| \leq 1$. Denote by $\omega^{+}(z)$ the harmonic measure of the segment $\{\operatorname{Re} z=-1 / 2\} \cap D^{+}$based at $z$ with respect to the domain $D^{+} \cap\{\operatorname{Re} z>-1 / 2\}$ and let $\gamma>1$ be chosen so that $\eta:=\sup _{|t| \leq 1} \omega(i t)<1 / \gamma<1$. By our hypothesis, $\rho^{+}(z) \geq 1-\varepsilon$ for $|z|$ close to 1 and $\geq \varepsilon^{\gamma}$ for $\operatorname{Re} z \geq-1 / 2$ and $|z|<1$. By the Maximum Principle we conclude that

$$
u^{+}(i t) \leq \log \frac{1}{1-\varepsilon}+\alpha \log \frac{1}{\varepsilon},
$$

where $\alpha=\gamma \eta<1$. Thus, there is $0<\varepsilon_{0}<1$ so that $u^{+}(i t)<\log \frac{1}{\varepsilon}$ for all $0<\varepsilon<\varepsilon_{0}$ and $|t| \leq 1$. But, by the symmetry mentioned above that $u^{ \pm}$satisfy, this clearly means that $f$ has a holomorphic extension to a domain $\tilde{\Omega}$ that contains a graph of a continuous function to which Chirka's Theorem can be applied. This finishes the proof.

\section{Proof of Theorem 2}

For the proof of Theorem 2 we will use Bharali's approach [2] to the generalization of the Chirka extension theorem. Let $a_{0}, \ldots, a_{n-1}$ be continuous functions on $\bar{\Delta}$ which define the continuous variety

$$
\Sigma=\left\{(z, w) \in \bar{\Delta} \times \mathbb{C} ; w^{n}+a_{n-1}(z) w^{n-1}+\cdots+a_{0}(z)=0\right\}
$$

over $\bar{\Delta}$. The mapping $\varphi: \bar{\Delta} \rightarrow \mathbb{C}^{n+1}$,

$$
\varphi: z \longmapsto\left(z, a_{0}(z), \ldots, a_{n-1}(z)\right),
$$

defines a continuous disc in $\mathbb{C}^{n+1}$. Any perturbation

$$
\widetilde{\varphi}: z \longmapsto\left(z, \widetilde{a}_{0}(z), \ldots, \widetilde{a}_{n-1}(z)\right)
$$


of this disc within the class of continuous functions on $\bar{\Delta}$ defines a new continuous variety over $\bar{\Delta}$,

$$
\widetilde{\Sigma}=\left\{(z, w) \in \bar{\Delta} \times \mathbb{C} ; w^{n}+\widetilde{a}_{n-1}(z) w^{n-1}+\cdots+\widetilde{a}_{0}(z)=0\right\},
$$

which is a perturbation of $\Sigma$.

For $\varepsilon>0$ let

$$
U(\varepsilon)=\bigcup_{(z, w) \in \Sigma}(\{z\} \times \overline{\Delta(w, \varepsilon)})
$$

be a closed neighbourhood of $\Sigma$ in $\bar{\Delta} \times \mathbb{C}$ and similarly let

$$
V(\varepsilon)=\bigcup_{z \in \bar{\Delta}}\left(\{z\} \times \overline{\Delta\left(a_{0}(z), \varepsilon\right)} \times \cdots \times \overline{\Delta\left(a_{n-1}(z), \varepsilon\right)}\right)
$$

be a closed neighbourhood of the disc $\varphi(\bar{\Delta})$ in $\bar{\Delta} \times \mathbb{C}^{n}$. Then for every $\varepsilon>0$ there exists $\delta>0$ such that for every continuous disc

$$
\widetilde{\varphi}: z \longmapsto\left(z, \widetilde{a}_{0}(z), \ldots, \widetilde{a}_{n-1}(z)\right),
$$

the condition

$$
\left(z, \widetilde{a}_{0}(z), \ldots, \widetilde{a}_{n-1}(z)\right) \in V(\delta) \quad \text { for some } z \in \bar{\Delta}
$$

implies that every point $(z, w) \in \widetilde{\Sigma}$ lies in $U(\varepsilon)$; that is, whenever a part of the image of the disc $\widetilde{\varphi}(\bar{\Delta})$ lies inside $V(\delta)$, the corresponding part of the continuous variety $\widetilde{\Sigma}$ lies within $U(\varepsilon)$.

Hence, if we can perturb the disc $\varphi$ within a continuous family of continuous discs $\varphi_{t}, t \in[0,1]$, on $\bar{\Delta}$ in such a way that $\varphi_{0}=\varphi, \varphi_{1}$ is a holomorphic disc and each $\varphi_{t}$ is holomorphic on the set of points which are mapped outside a closed neighbourhood of the disc $\varphi(\Delta)$, then we can perturb the continuous variety $\Sigma$ through a continuous family $\Sigma_{t}, t \in[0,1]$, of continuous varieties over $\bar{\Delta}$ in such a way that $\Sigma_{0}=\Sigma, \Sigma_{1}$ is an analytic variety and each continuous variety $\Sigma_{t}$ is such that it is holomorphic outside a given closed neighbourhood of $\Sigma$. Then, using analytic continuation [6], one can extend a given holomorphic function $f$ in a connected neighbourhood of the set $K=\Sigma \cup(\partial \Delta \times \mathbb{C})$ to a neighbourhood of $K_{1}=\Sigma_{1} \cup(\partial \Delta \times \mathbb{C})$. By Hartogs' extension theorem we conclude that $f$ extends holomorphically to a neighbourhood of $\bar{\Delta} \times \mathbb{C}$.

Remark 3. The continuation of $f$ given by [6] does not a priori rule out multivaluedness. However, in a tubular neighbourhood $\mathcal{V}$ of $\partial \Delta_{z} \times \mathbb{C}_{w}$, the family $\left\{\Sigma_{t} \mid \mathcal{V}\right\}$ evolves in the direction of $\mathbb{C}_{w}$, which is simply connected. Thus, this extension agrees with $f$ on $\mathcal{V}$. Hence they agree everywhere where both are defined.

We then seek for a perturbation of the disc $\varphi(\bar{\Delta})$ within a continuous family of continuous discs $\varphi_{t}, t \in[0,1]$, on $\bar{\Delta}$ in such a way that $\varphi_{0}=\varphi, \varphi_{1}$ is a holomorphic disc and each $\varphi_{t}$ is holomorphic on the set of points which are mapped outside a closed neighbourhood of $\varphi(\bar{\Delta})$. This has basically already been done in Bharali's paper [2, and we include the necessary argument for the sake of completeness.

First let us observe that without loss of generality we may assume that $a_{j}(z)=$ $P_{j}(z, \bar{z}), j=0, \ldots, n-1$, for some holomorphic polynomials $P_{j}(z, \xi)$ in two complex variables. The mapping

$$
(z, \xi) \in \bar{\Delta} \times \mathbb{C} \longrightarrow\left(z, P_{0}(z, \xi), \ldots, P_{n-1}(z, \xi)\right) \in \bar{\Delta} \times \mathbb{C}^{n}
$$


is holomorphic and maps the maximal real disc $\mathcal{R}=\{(z, \bar{z}) \in \bar{\Delta} \times \mathbb{C}\}$ into $\varphi(\bar{\Delta})$ and a neighbourhood of $\mathcal{R}$ in $\bar{\Delta} \times \mathbb{C}$ into a neighbourhood of $\varphi(\bar{\Delta})$ in $\bar{\Delta} \times \mathbb{C}^{n}$. In the proof of Lemma 1.4 in [2], Bharali constructed an explicit family of holomorphic discs in $\bar{\Delta} \times \mathbb{C}$ with boundaries in $\mathcal{R}$ and $\partial \Delta \times \mathbb{C}$. For $t \in[-1,1]$, let

$$
\mathcal{A}_{t}(z)=(z, 2 t-z) \text {. }
$$

For $t \in[0,1]$ the $\operatorname{disc} \mathcal{A}_{t}$ is defined on

$$
\mathcal{D}_{t}^{+}=\left\{x+i y ; t \leq x \leq 1, x^{2}+y^{2} \leq 1\right\}
$$

while for $t \in[-1,0]$ it is defined on

$$
\mathcal{D}_{t}^{-}=\left\{x+i y ;-1 \leq x \leq t, x^{2}+y^{2} \leq 1\right\} .
$$

For each $t \in[0,1]$ we define a continuous disc $\Phi_{t}$ on $\bar{\Delta}$ as

$$
\Phi_{t}(z)=\left\{\begin{array}{l}
\mathcal{A}_{t-1}(z) \text { for } z \in \mathcal{D}_{t-1}^{-}, \\
\mathcal{A}_{1-t}(z) \text { for } z \in \mathcal{D}_{1-t}^{+}, \\
(z, \bar{z}) \text { elsewhere on } \bar{\Delta}
\end{array}\right.
$$

Finally we define for $t \in[0,1]$,

$$
\varphi_{t}(z)=\left(z, P_{0}\left(\Phi_{t}(z)\right), \ldots, P_{n-1}\left(\Phi_{t}(z)\right)\right) .
$$

The discs $\left\{\varphi_{t}\right\}_{t \in[0,1]}$ form a continuous family of continuous discs on $\bar{\Delta}$ such that $\varphi_{0}=\varphi, \varphi_{1}$ is a holomorphic disc and each $\varphi_{t}$ is holomorphic on the set of points which are mapped outside a closed neighbourhood of the mapping $\varphi$. This finishes the proof of Theorem 2 ,

Remark 4. Another approach to the problem of extendability from a neighbourhood of the continuous variety $K=\Sigma \cup(\partial \Delta \times \mathbb{C})$ is also the following. Namely, one can prove that within a neighbourhood of $\Sigma$ there is a smooth variety $\widetilde{\Sigma}$, a bordered Riemann surface $S$, a proper holomorphic function $a: S \rightarrow \Delta$ and a smooth function $\phi: \bar{S} \rightarrow \mathbb{C}$ such that

$$
(a, \phi): \bar{S} \longrightarrow \bar{\Delta} \times \mathbb{C}
$$

is a smooth embedding whose image is $\widetilde{\Sigma}$. Using this fact, one is left to consider a similar problem as Chirka above the bordered Riemann surface $S$ : Let $S$ be a bordered Riemann surface and let $\phi: S \rightarrow \bar{\Delta}$ be a continuous function on $S$. Does every function holomorphic in a connected neighbourhood of $\operatorname{graph}(\phi) \cup(\partial S \times \bar{\Delta})$ holomorphically extend to a neighbourhood of $S \times \bar{\Delta}$ ? If $S$ is a planar domain, that is, the genus of $S$ is 0 , then the same proof as Chirka's [4] works. The case of higher genus $g>0$ seems to be much harder and in generally open. In cases where the degree of the 'Weierstrass polynomial' is greater than or equal to the genus of (a uniformly small smooth perturbation of) $S$, the extension result follows from Theorem 12 in 9 .

For Chirka's technique to work, it is necessary to have sup norm control on the solution to a certain quasilinear $\bar{\partial}$-equation on $S$, something we do not have when $g>0$. Also, this is essentially the raison d'être for Bharali's class of functions in [2] to be that restrictive. An attempt to get such control via Riemann-Hilbert boundary value problems for quasilinear $\bar{\partial}$-equations on bordered Riemann surfaces yielded [3]. However the sup norm control one needs to conclude the proof of the generalization of Chirka's result over bordered Riemann surfaces with genus $g>0$ seems to be still unknown. 


\section{REFERENCES}

[1] D. E. Barrett and G. Bharali, The role of Fourier modes in extension theorems of HartogsChirka type, Math. Z. 249 (2005), 883-901. MR2126221 (2005m:32016)

[2] G. Bharali, Some generalizations of Chirka's extension theorem, Proc. Amer. Math. Soc. 129 (2001), 3665-3669. MR1860501 (2002i:32008)

[3] M. Cerne and M. Flores, Quasilinear d-equation on bordered Riemann surfaces, Math. Ann. 335 (2006), 379-403. MR2221118 (2007b:35247)

[4] E. M. Chirka, The generalized Hartogs lemma and the nonlinear $\bar{\partial}$-equation, Complex analysis in modern mathematics (Russian), 19-31, FAZIS, Moscow, 2001. MR.1833502 (2002k:32013)

[5] E. M. Chirka and J.-P. Rosay, Remarks on the proof of a generalized Hartogs lemma, Complex analysis and applications (Warsaw, 1997). Ann. Polon. Math. 70 (1998), 43-47. MR.1668715 (2000a:32071)

[6] E. M. Chirka and E. L. Stout, A Kontinuitätssatz, Topics in complex analysis (Warsaw, 1992), Banach Center Publ., 31, Polish Acad. Sci., Warsaw, 1995, 143-150. MR.1341384(96g:32026)

[7] F. Forstnerič, Stein domains in complex surfaces, J. Geom. Anal. 13 (2003), 77-94. MR.1967038 (2004c:32050)

[8] S. Ivashkovich and V. Shevchishin, Structure of the moduli space in a neighborhood of a cuspcurve and meromorphic hulls, Invent. Math. 136 (1999), 571-602. MR.1695206|(2001d:32035)

[9] S. Y. Nemirovski, Complex analysis and differential topology on complex surfaces (Russian), Uspekhi Mat. Nauk 54 (1999), 47-74; translation in Russian Math. Surveys 54 (1999), 729752. MR:1741278 (2000k:32018)

[10] R. M. Range, Holomorphic functions and integral representations in several complex variables, Graduate Texts in Mathematics, 108, Springer-Verlag, New York, 1986. MR847923 (87i:32001)

[11] J.-P. Rosay, A counterexample related to Hartogs' phenomenon (a question by E. Chirka), Michigan Math. J. 45 (1998), 529-535. MR1653267(2000a:32070)

Department of Mathematics, University of Ljubljana, Jadranska 19, 1111 Ljubljana, SLOVENIA

E-mail address: miran.cerne@fmf.uni-lj.si

Department of Mathematics, University of La Laguna, 38771 La Laguna, Tenerife, SPAIN

E-mail address: mflores@ull.es 